\title{
Analytical Expressions for Lightning Electromagnetic Fields with Arbitrary Channel Base Current. Part II: Validation and Computational Performance
}

\author{
Daniele Mestriner, Member IEEE, Massimo Brignone, Member IEEE, Renato Procopio, Senior \\ Member IEEE Mansueto Rossi, Member IEEE, Federico Delfino Member IEEE, Farhad Rachidi, \\ Fellow IEEE and Marcos Rubinstein, Fellow IEEE
}

\begin{abstract}
The paper provides the implementation procedure, the validation and some considerations on the computational efforts of the developed analytical expressions for the lightning electromagnetic fields presented in the companion paper. The validation is presented with different configurations in terms of channel-base current, ground conductivity and distance to the lightning channel, comparing the obtained results with the numerical integration of the classical formulas. The comparative analysis shows a perfect agreement between the proposed analytical approach and reference numerical simulations. Moreover, the computational effort of the proposed method is discussed, focusing the attention on the choice of the points in which the channel has to be divided in order to maximize the CPU time savings without losing accuracy.
\end{abstract}

Index Terms - Lightning electromagnetic fields, Channel base current, Engineering models

\section{INTRODUCTION}

$\mathrm{O}$ ne of the most important causes of damages in distribution systems are lightning-induced voltages. As pointed out in Part I, their evaluation has been addressed by many researchers in the last years. It is important to note that all the proposed models for the computation of induced voltages rely on the knowledge of the electromagnetic fields. In the literature, their evaluation is usually achieved in two steps: 1) the electromagnetic fields in the presence of a perfectly conducting ground are computed assuming a vertical lightning channel [1-3], and 2) the effect of the ground conductivity is taken into account with the Cooray-Rubinstein formula [4, 5].

Massimo Brignone, Renato Procopio, Daniele Mestriner, Mansueto Rossi and Federico Delfino and are with the Naval ICT and Electrical Engineering Department (DITEN), University of Genoa, Via Opera Pia 11a, I-16145 Genova, Italy (e-mail: massimo.brignone@unige.it, renato.procopio@ unige.it, daniele.mestriner@edu.unige.it, mansueto.rossi@unige.it, federico.delfino@unige.it). Farhad Rachidi is with the EMC Group, Swiss Federal Institute of Technology, EPFL-STI-LRE, Station 11, CH-1015 Lausanne, Switzerland (email: farhad.rachidi@epfl.ch). Marcos Rubinstein is with the University of Applied Sciences Western Switzerland, Yverdon-lesBains 1401, Switzerland (e-mail: marcos.rubinstein@heighvd.ch)
However, when dealing with lightning-induced voltages adopting a statistical approach (which is the case of the lightning performance evaluation), the solution of the aforementioned two-steps approach is not an easy task, especially in terms of the high computational effort caused by the need of evaluating the fields at many different distances from the lightning strike. As noted in Part I, the main solutions have been proposed in [6] and [7] relying on either analytical or numerical approaches, respectively. However, the analytical approach [6] is valid only under some assumptions (perfectly conducting ground and trapezoidal channel base current), while the numerical one (that builds up a field database and then obtains the required fields by means of linear interpolation [7]) has the drawback of a high computational effort, especially when the effect of the current front time must be accounted for. In this context, a new approach has been proposed in Part I. The method is based on analytical formulas for lightning electromagnetic fields generated by an arbitrarily shaped channel base current over a perfectly conducting (for all the components of the electric and magnetic fields) or a lossy ground (for the radial component of the electric field). This paper is mainly divided in three parts: In the first (Section II), a step-by-step procedure that can help the reader to implement the proposed method is provided. In the second part (Section III), the validation of the proposed method against the reference numerical approach [1] is presented. In the third part (Section IV), some considerations on the computational effort are provided, focusing on the best solution in terms of computational time and accuracy. Finally, conclusions are drawn in Section V.

\section{IMPLEMENTATION OF THE PROPOSED APPROACH}

In this section, a step-by-step implementation procedure is proposed.

The main step is to provide a discretized version of the function $f(\eta)=\sqrt{1+\eta^{2}}$ via the function $g$ (Eq. (A1) of Part I). Let us consider the geometry presented in Fig. 1. In order to do 
that, remembering that $\eta=\left(z^{\prime}-z\right) / r$, one has to:

1. Choose the height $z$ and the distance $r$ from the lightning strike where the fields have to be computed (i.e. the coordinates of the observation point $Q$ );

2. Evaluate the quantity

$$
\eta_{\max }=A=\frac{H-z}{r}
$$

where $H$ is the height of the lightning channel.

3. Choose the number of intervals $N$ into which the lightning channel is divided.

4. Evaluate $\alpha_{j}, a_{j}$ and $b_{j}$ (with $j=1, \ldots, N+1$ ) according to the procedure presented in the Appendix of Part I (a detailed analysis on possible choices for the values of $\eta_{\max }$ and $N$ is proposed in Section $\mathrm{V}$ ).

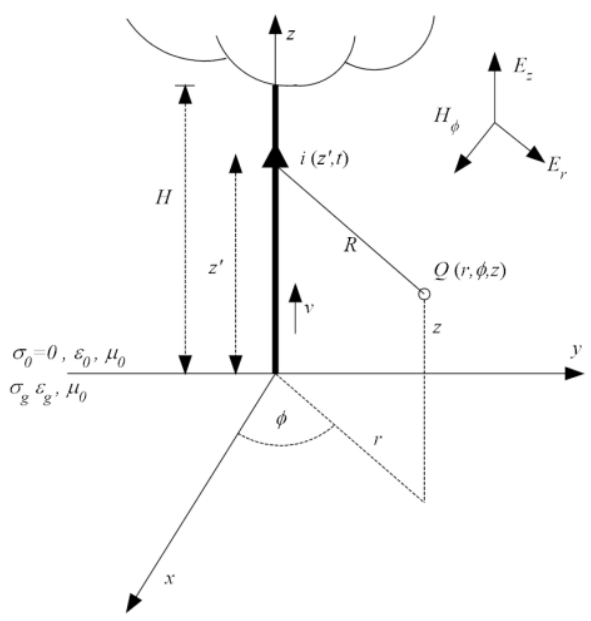

Fig. 1 Geometry of the problem

Once the aforementioned quantities are determined, one can calculate the magnetic field $H_{\varphi}^{i d}$ for a perfectly-conducting ground according to the following scheme

1. Find the index $j^{*} \in\{1, \ldots, N\}$ such that

$$
-\alpha_{j^{*}} \leq-z / r \leq-\alpha_{j^{*}-1}
$$

2. Fix the time $t_{*}$ before that the fields at $Q$ are zero

$$
t_{*}=\frac{b_{j^{*}}}{c_{0}} r+\frac{a_{j^{*}}}{c_{0}} z
$$

3. Fix the time instants $t_{j}^{i}$ for which the dipole at point $-\alpha_{j}$ contributes to the fields at $Q$ ( $v$ is the current propagation speed and $c_{0}$ is the speed of light in vacuum).

$$
t_{j}^{i}=\left(\frac{a_{i} \alpha_{j}+b_{j}}{c_{0}}+\frac{\alpha_{j}}{v}\right) r-\frac{1}{v} z
$$

4. Compute the time instants $t_{0}^{s, 2}=t_{0}^{s, 1}$ at which the dipole placed at $x=0$ starts contributing to the field

$$
t_{0}^{s, 1}=t_{0}^{s, 2}=\frac{1}{c_{0}} r+\frac{1}{v} z
$$

5. Compute the time instants $t_{j}^{s, 1}$ (for $j=1, \ldots, j^{*}-1$ and $j^{*}>1$ ) and $t_{j}^{s, 2}$ (for $j=1, \ldots, N$ ) necessary for the fields generated at points $-\alpha_{j}$ and $\alpha_{j}$ of the source to reach point $Q$

$$
\begin{aligned}
& t_{j}^{s, 1}=\left(\frac{a_{j} \alpha_{j}+b_{j}}{c_{0}}-\frac{\alpha_{j}}{v}\right) r+\frac{1}{v} z \\
& t_{j}^{s, 2}=\left(\frac{a_{j} \alpha_{j}+b_{j}}{c_{0}}+\frac{\alpha_{j}}{v}\right) r+\frac{1}{v} z
\end{aligned}
$$

6. Evaluate the magnetic field according to equations (5), (6),(13),(19),(20) of Part I.

Once the evaluation of the magnetic field has been performed, the electric field components or a perfectlyconducting ground can be easily calculated according to equations (21)-(26) of Part I.

Finally, the influence of the ground conductivity on the radial electric field can be accounted for by using the proposed time domain analytical expression of the Cooray-Rubinstein formula (equations (29)-(37) in the Part I paper), based on the expression of the channel base current as a sum of exponential terms (see [8] for details).

\section{VALIDATION OF THE PROPOSED APPROACH}

The proposed method will be compared with the results of the so-called "numerical method", corresponding to the numerical integration of i) the classical formulas for the ideal fields [1-3], and ii) the time domain expression for the CoorayRubinstein formula described in [9] (which is a compromise between computational time and accuracy, avoiding a numerical convolution but maintaining the approximation error always lower than $0.01 \%$ ) using a time step of $10 \mathrm{~ns}$ [10]. Note that, even if it requires a high computational effort, the numerical integration [1] has been used as a benchmark because other works $([11,12])$ provide closed-form expressions for step excitations and require a numerical, time consuming, convolution if an arbitrary channel-base expression is taken into account and if the fields are computed at heights different from $\mathrm{z}=0$. In the following, the comparison will take into account different ground conductivities and different expressions for the channel base current (Fig. 2). For each of the proposed configurations, the electromagnetic fields will be evaluated at a height of $10 \mathrm{~m}$ and at three different distances from the channel, i.e. $50 \mathrm{~m}, 200 \mathrm{~m}$ and $2000 \mathrm{~m}$. The details of each test are summarized in TABLE 1.

The height of the return stroke channel is assumed to be 8 $\mathrm{km}$, while the return stroke propagation velocity is $c_{0} / 2\left(c_{0}\right.$ is the speed of light in vacuum).

TABLE 1 Details of the tests. "First (Subsequent)" indicates that the channel base current follows the Heidler's formula with parameters typical of first (subsequent) stroke currents; "Measured" means that the channel base current used is taken from a set of Lightning Measurements obtained at Mount Säntis Station in Switzerland. The ground conductivity is denoted by $\sigma$, and PEC stays for Perfect Conducting Ground, as usual.

\begin{tabular}{|c|c|c|c|}
\hline Test & $\boldsymbol{r}[\mathbf{m}]$ & Channel base current & $\boldsymbol{\sigma}[\mathbf{m S} / \mathbf{m}]$ \\
\hline T1-A & 50 & first return stroke [13] & PEC \\
\hline T1-B & 200 & first return stroke & PEC \\
\hline
\end{tabular}




\begin{tabular}{|c|c|c|c|}
\hline T1-C & 2000 & first return stroke & PEC \\
\hline T2-A & 50 & subsequent return stroke [13, 14] & PEC \\
\hline T2-B & 200 & subsequent return stroke & PEC \\
\hline T2-C & 2000 & subsequent return stroke & PEC \\
\hline T3-A & 50 & measured subsequent stroke [8] & PEC \\
\hline T3-B & 200 & measured subsequent stroke & PEC \\
\hline T3-C & 2000 & measured subsequent stroke & PEC \\
\hline T4-A & 50 & subsequent return stroke & 1 \\
\hline T4-B & 200 & subsequent return stroke & 1 \\
\hline T4-C & 2000 & subsequent return stroke & 1 \\
\hline T5-A & 50 & subsequent return stroke & 10 \\
\hline T5-B & 200 & subsequent return stroke & 10 \\
\hline T5-C & 2000 & subsequent return stroke & 10 \\
\hline
\end{tabular}
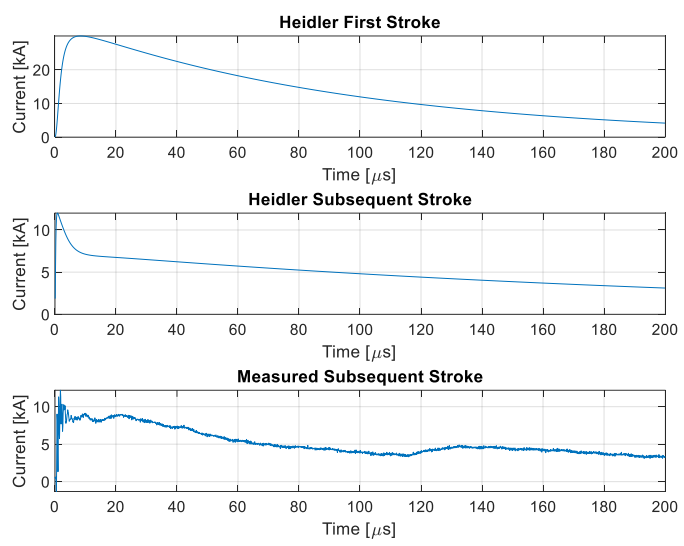

Fig. 2 Considered current waveforms. (a) Typical first return stroke. (b) Typical subsequent return stroke. (c) Measured subsequent return stroke [8]

Tests T1, T2 and T3 aim at validating the developed analytical formulas for ideal fields with different channel base currents, while tests $\mathrm{T} 4$ and $\mathrm{T} 5$ are performed to show the accuracy of the proposed expression of the Cooray-Rubinstein formula.

Let us select a number of discretization intervals $\mathrm{N}=50$. We will discuss in Section IV the effect of the choice of $\mathrm{N}$. Furthermore, the values of ${ }_{\max }$ for $r=50,200$ and $2000 \mathrm{~m}$ are given by

$$
\eta_{\max }=\frac{H-z}{r}=\frac{8000-10}{r}= \begin{cases}159.80 & r=50 \mathrm{~m} \\ 39.95 & r=200 \mathrm{~m} \\ 3.99 & r=2000 \mathrm{~m}\end{cases}
$$

According to the procedure described in the Appendix of Part I, the discretization points $\alpha_{j}$ (with $j=1, \ldots, N+1$ ) are presented in Fig. 3 for the three selected values of $r$. The comparison results for the considered test cases are presented in Figs. 4 to 18. As can be seen from the figures, there is an excellent agreement between the proposed approach and the numerical calculation for all the considered cases, with some slight deviations in the case of a measured current waveform, probably related to the presence of noise in the current waveform, even if it has been previously filtered with a lowpass filter.

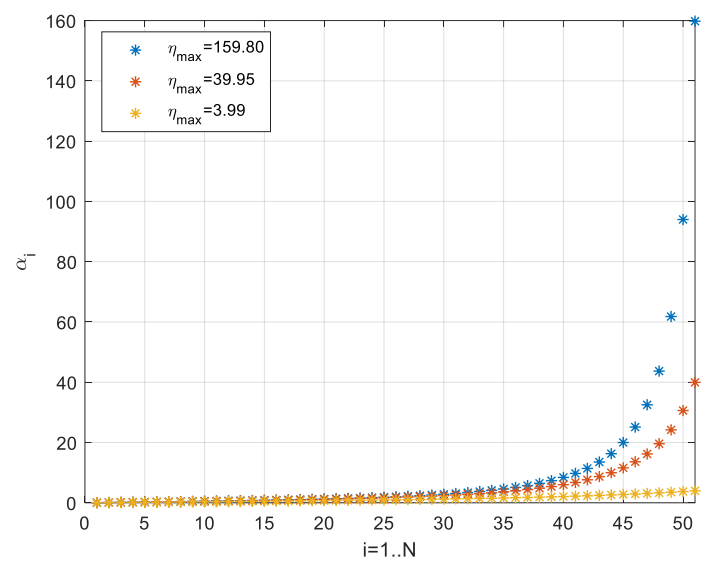

Fig. 3 Discretizing points of $f$ for three different values of $\mathrm{x}_{\max }$, corresponding to horizontal distances $r=50,200$ and $2000 \mathrm{~m}$. The height of the channel is $8 \mathrm{~km}$ and the height of the observation point $10 \mathrm{~m}$.

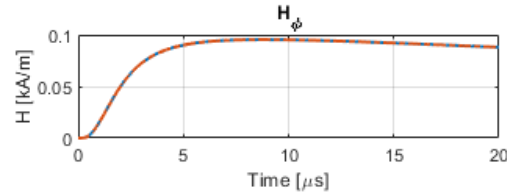

- Proposed Approach

$E_{r}$

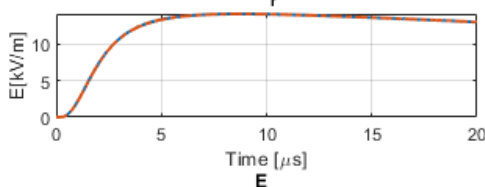

- Proposed Approach ----- Numerical Approach

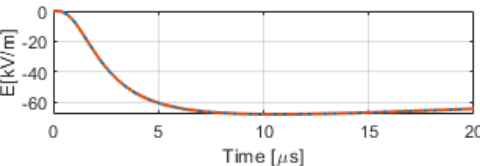

- Proposed Approach ----- Numerical Approach

Fig. 4 Test T1-A. First return stroke (Heidler current). The distance to the channel is $50 \mathrm{~m}$ and the ground is assumed to be perfectly conducting.
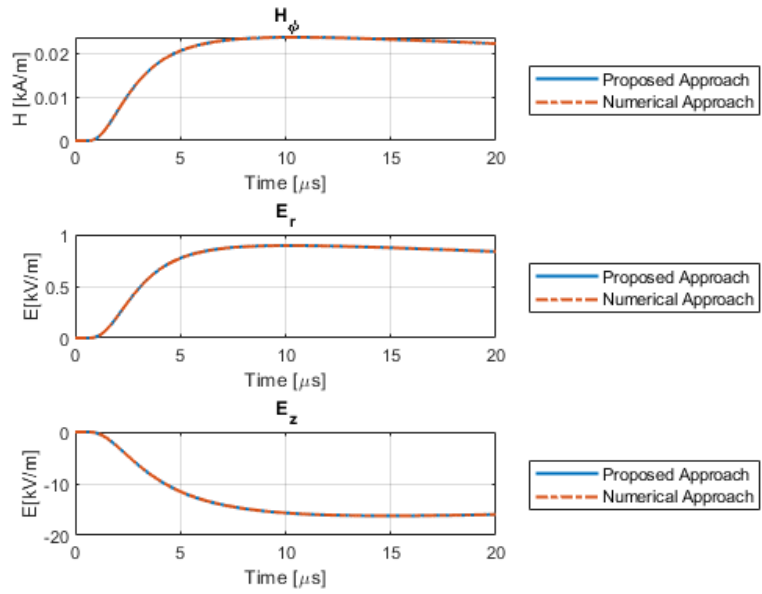

Fig. 5 Test T1-B. First return stroke (Heidler current). The distance to the channel is $200 \mathrm{~m}$ and the ground is assumed to be perfectly conducting. 


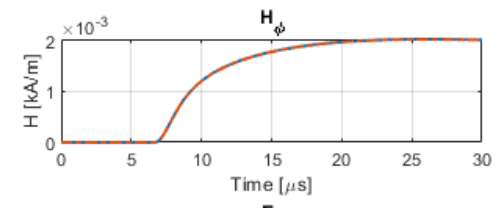

- Proposed Approach
----- Numerical Approach

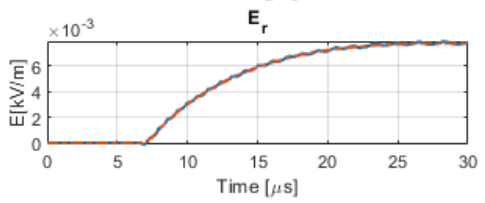

Proposed Approach

$E_{z}$

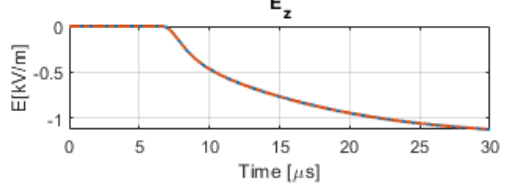
- Proposed Approach

Fig. 6 Test T1-C. First return stroke (Heidler current). The distance to the channel is $2000 \mathrm{~m}$ and the ground is assumed to be perfectly conducting.
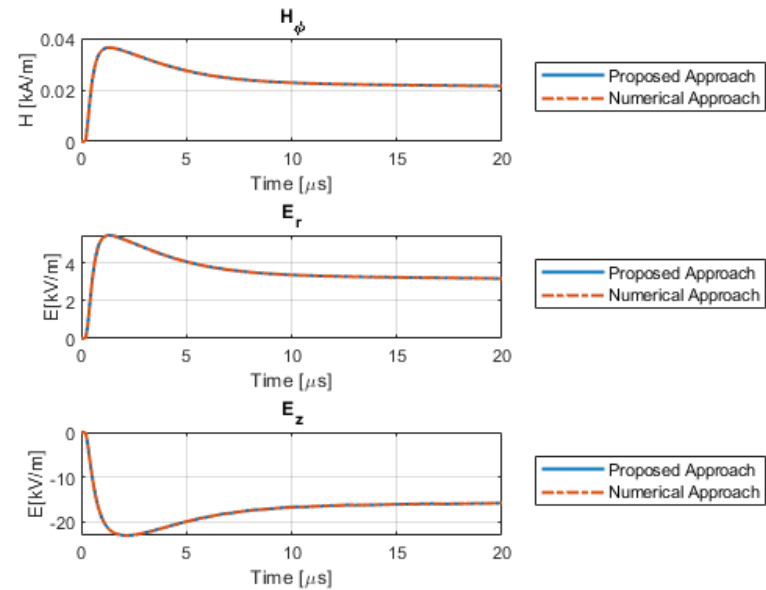

- Proposed Approach -----Numerical Approach

Fig. 7 Test T2-A. Subsequent return stroke current. The distance to the channel is $50 \mathrm{~m}$ and the ground is assumed to be perfectly conducting.
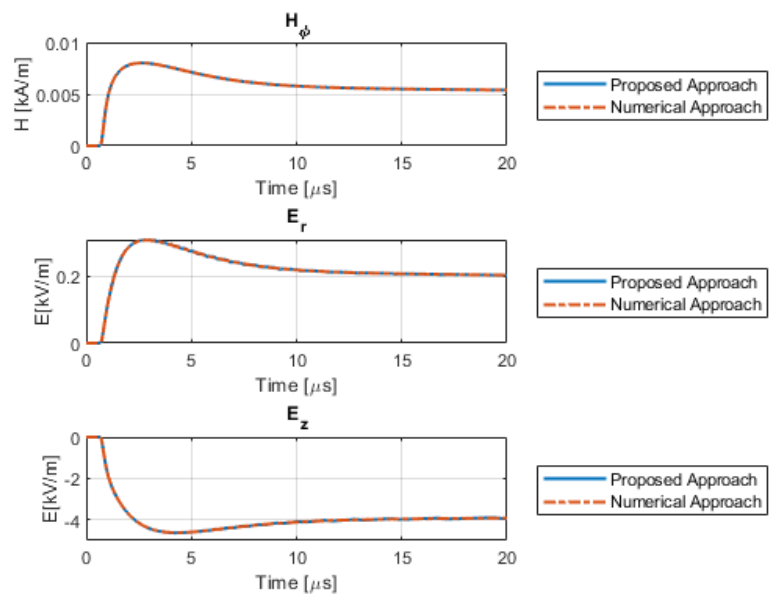

----- Numerical Approach

Fig. 8 Test T2-B. Subsequent return stroke current. The distance to the channel is $200 \mathrm{~m}$ and the ground is assumed to be perfectly conducting.
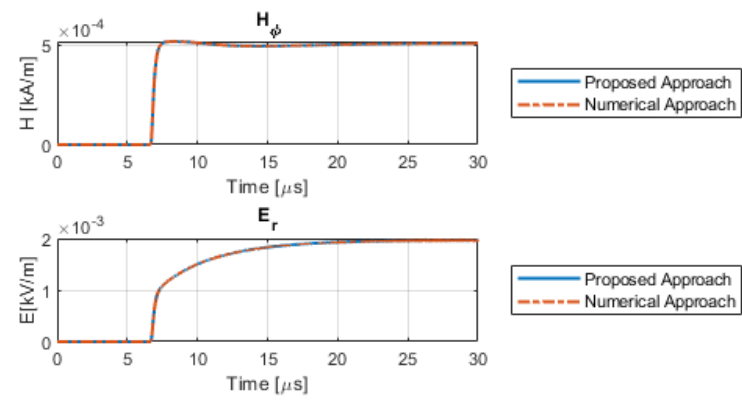

- Proposed Approach ----- Numerical Approach

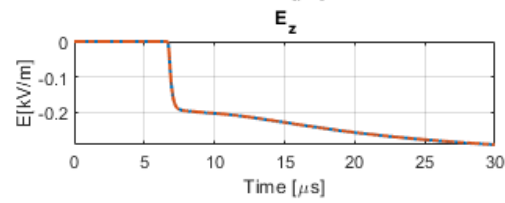

Proposed Approach ----- Numerical Approach

Fig. 9 Test T2-C. Subsequent return stroke current. The distance to the channel is $2000 \mathrm{~m}$ and the ground is assumed to be perfectly conducting.

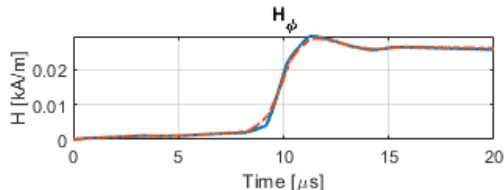

- Proposed Approach
---- Numerical Approach

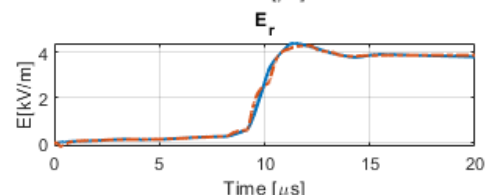

- Proposed Approach ----- Numerical Approach

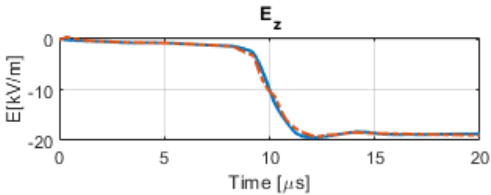

- Proposed Approach ----- Numerical Approach

Fig. 10 Test T3-A. Measured return stroke current. The distance to the channel is $50 \mathrm{~m}$ and the ground is assumed to be perfectly conducting.
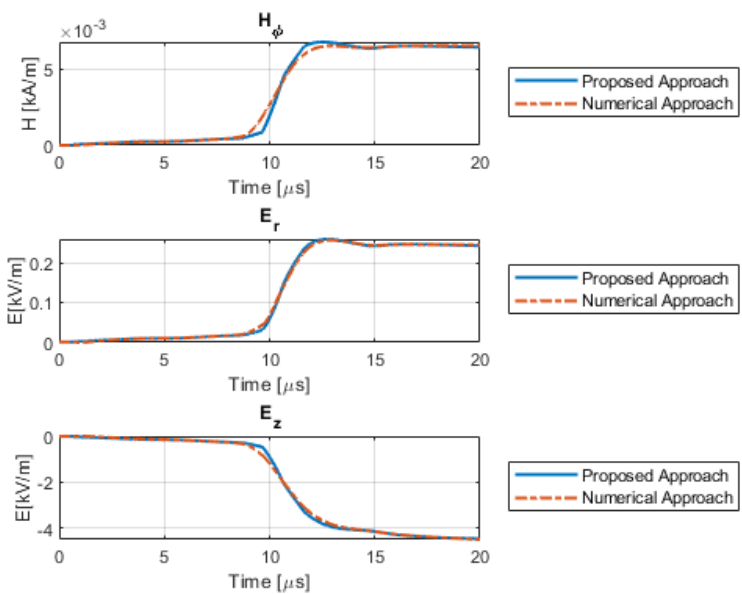

Proposed Approach -----Numerical Approach

Fig. 11 Test T3-B. Measured return stroke current. The distance to the channel is $200 \mathrm{~m}$ and the ground is assumed to be perfectly conducting. 

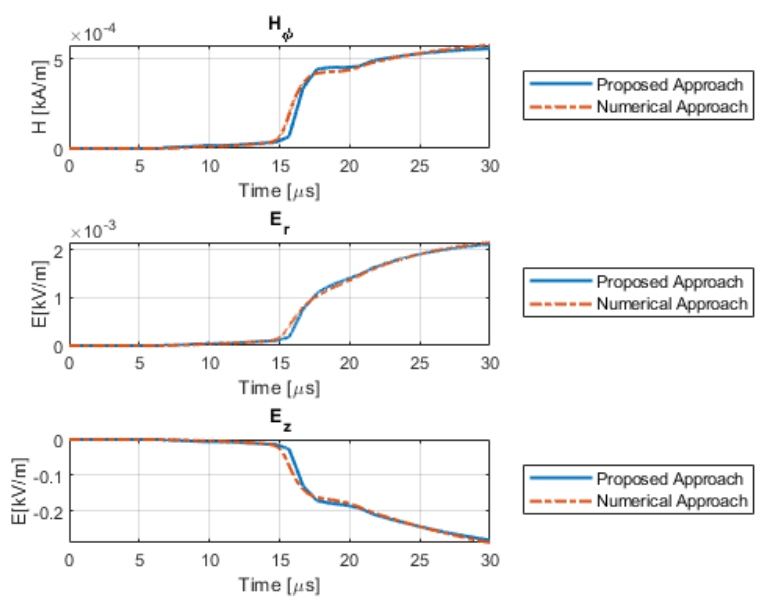

Fig. 12 Test T3-C. Measured return stroke current. The distance to the channel is $2000 \mathrm{~m}$ and the ground is assumed to be perfectly conducting.

$E_{r}$

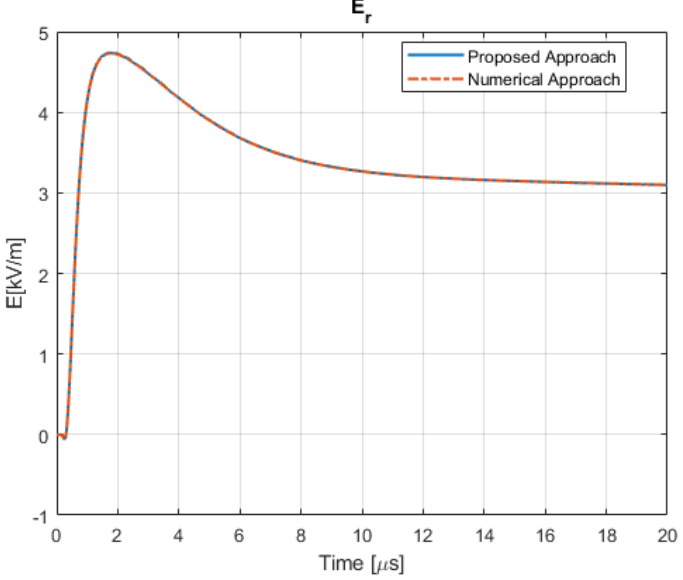

Fig. 13 Test T4-A. Subsequent return stroke. The distance to the channel is 50 $\mathrm{m}$ and the ground is assumed to have a $1 \mathrm{mS} / \mathrm{m}$ conductivity.

$E_{\mathrm{r}}$

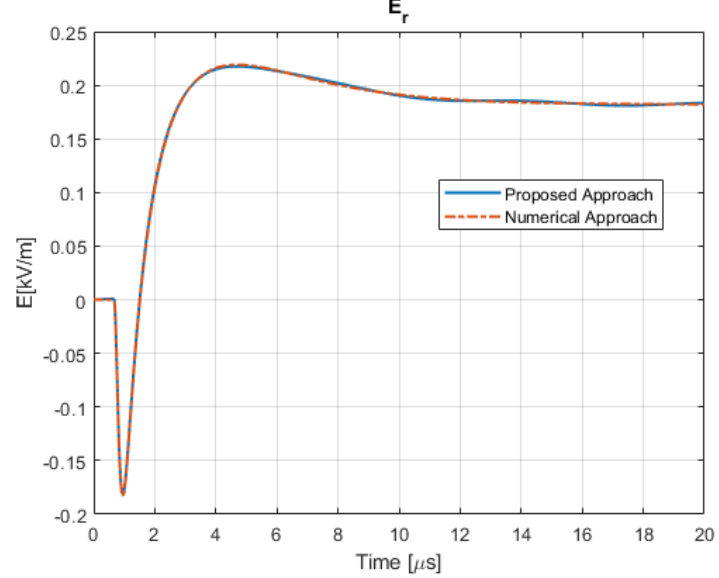

Fig. 14 Test T4-B. Subsequent return stroke. The distance to the channel is 200 $\mathrm{m}$ and the ground is assumed to have a $1 \mathrm{mS} / \mathrm{m}$ conductivity.

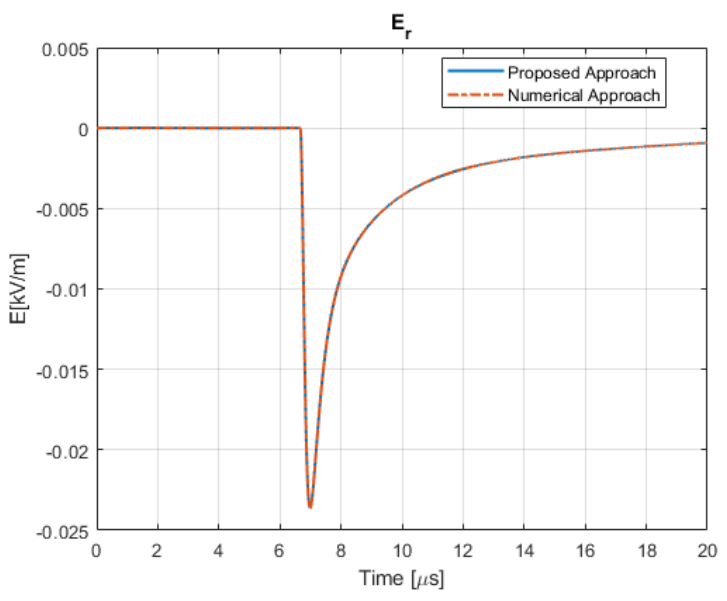

Fig. 15 Test T4-C. Subsequent return stroke. The distance to the channel is 2000 $\mathrm{m}$ and the ground is assumed to have a $1 \mathrm{mS} / \mathrm{m}$ conductivity.

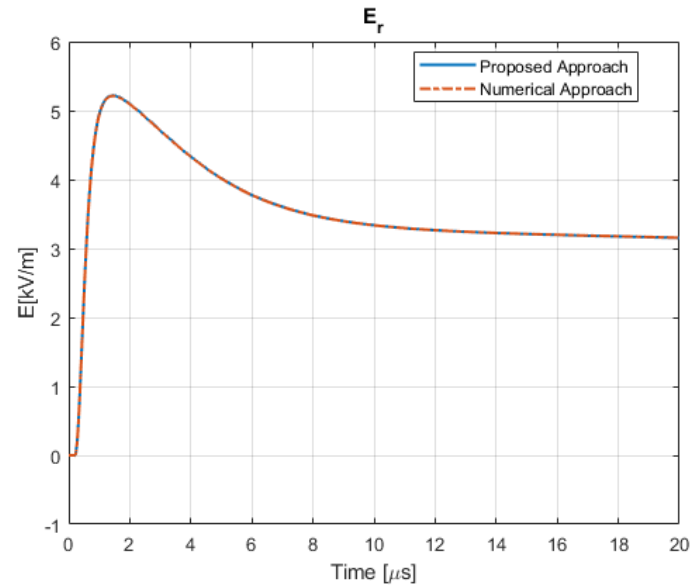

Fig. 16 Test T5-A. Subsequent return stroke. The distance to the channel is 50 $\mathrm{m}$ and the ground is assumed to have a $10 \mathrm{mS} / \mathrm{m}$ conductivity.

$E_{r}$

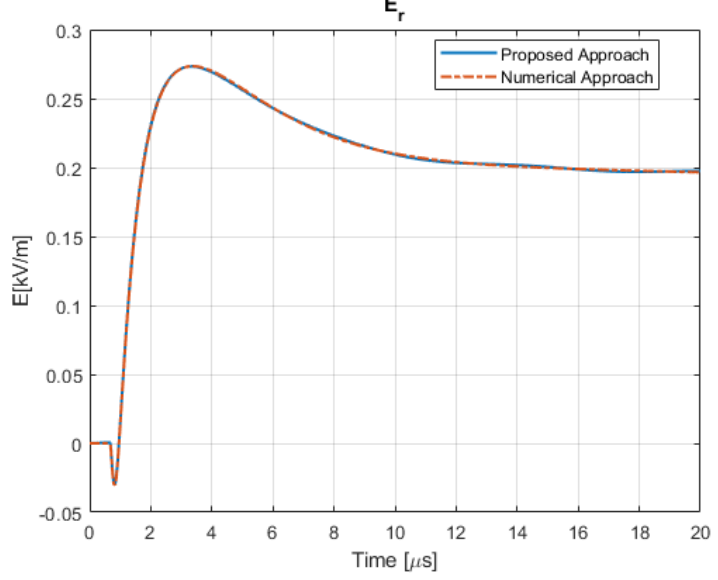

Fig. 17 Test T5-B. Subsequent return stroke. The distance to the channel is 200 $\mathrm{m}$ and the ground is assumed to have a $10 \mathrm{mS} / \mathrm{m}$ conductivity. 


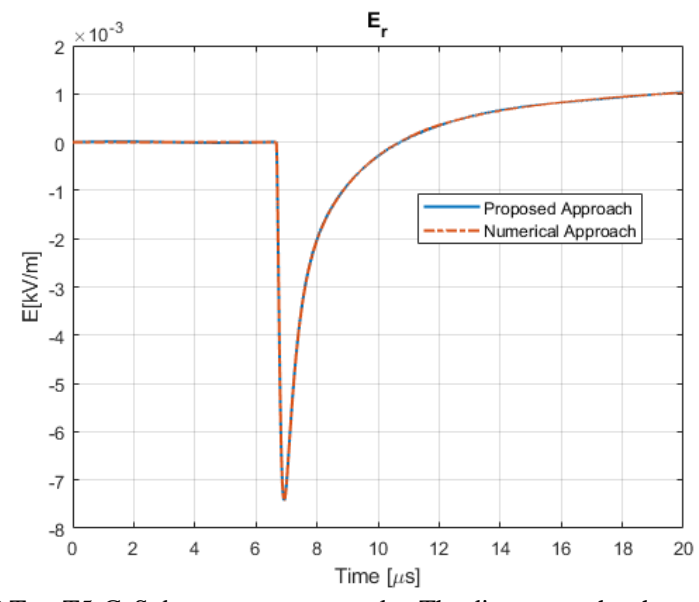

Fig. 18 Test T5-C. Subsequent return stroke. The distance to the channel is 2000 $\mathrm{m}$ and the ground is assumed to have a $10 \mathrm{mS} / \mathrm{m}$ conductivity.

\section{COMPUTATIONAL EFFORT OF THE PROPOSED METHOD}

In this section, some considerations on the computational effort of the proposed method are given. It is important to notice that the computational effort is strictly related to the discretization of the function $f$. As highlighted in Section II, two main factors can affect it: the choice of the maximum value $x_{\max }$ and the number $N$ of intervals into which the function is discretized.

In the first part of this section (subsection $\mathrm{A}$ ), the focus will be on the choice of $\eta_{\max }$, while in subsection $\mathrm{B}$, the results of the proposed method for different values of $N$ will be analyzed and discussed, showing the strength of the proposed method in terms of reduction of the computational time.

\section{A. The choice of $\eta_{\max }$}

As highlighted in Section II, the value of $\eta_{\max }$ changes according to the coordinates of point $Q$. This means that, in principle, the discretization of $f$ changes according to $r$ and $z$. This represents a disadvantage, especially when dealing with multiple field calculations (e.g., in any field-to-line coupling problem) because it would imply solving an optimization problem for each horizontal distance and height.

This problem can be overcome by setting $\eta_{\max }$ to the maximum value it can assume in the domain of interest of the observation point $Q$. From (1) it is easy to notice that, for an assigned return stroke channel height $H$, it corresponds to the minimum horizontal distance $r_{\min }$ and to the minimum height $z_{\min }$. In the case of induced effects of lightning on power lines, usually a distance from the striking point closer than $r_{\text {min }}=20 \mathrm{~m}$ is never considered because this would lead to a direct event and the height of a power line is never lower than $z_{\min }=5 \mathrm{~m}$. With this choice, for all the other observation points, $\eta_{\max }$ corresponds to a point beyond the channel height, which is of no use for the field calculations. This means that, in principle, for all the other observation points, the points $\alpha_{j}$ do not optimize the objective function defined in the companion paper. However, in what follows, it is shown that such error has a negligible impact on the final result.

Let us reconsider Fig. 3. Despite the different values of $\eta_{\max }$ (i.e., 159.80, 39.95 and 3.99), it can be noticed that, in each case, the optimization procedure sets the majority of the discretizing points close to $\eta=0$. This can be well explained by the graph of $f$ (Fig. 19), which exhibits the maximum rate of change of its first derivative in the neighborhood of the origin, requiring there a higher number of secants to obtain a proper fitting with linear functions.

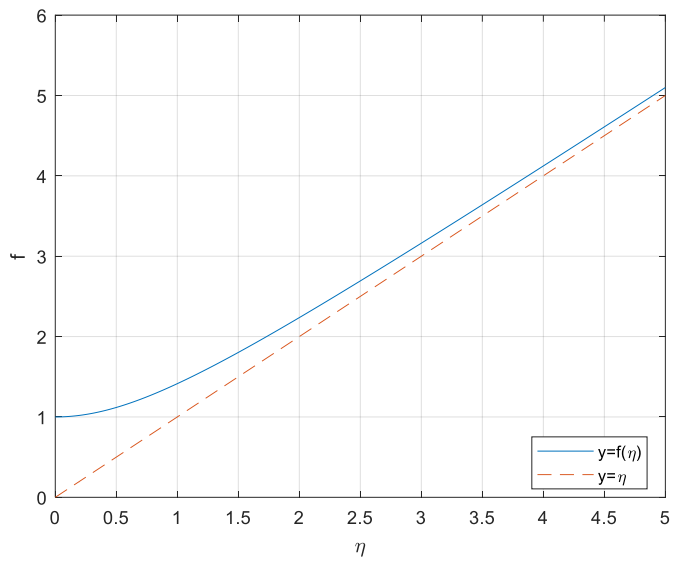

Fig. 19 Graph of the function $\mathrm{f}$

To give further evidence of this concept, let us set $\eta_{\max }=\left(H-z_{\min }\right) / r_{\min }=399.75$. The optimization procedure leads to the discretizing points plotted with the purple dots in Fig. 20, which are not so different from the other ones, confirming the aforementioned considerations.

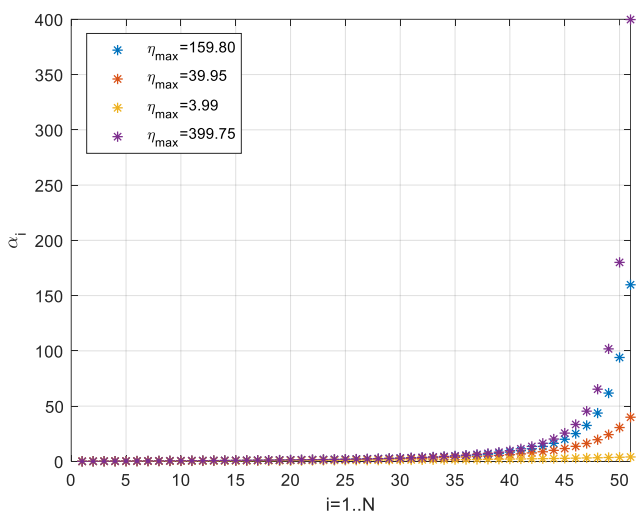

Fig. 20 Discretizing points for $\mathrm{N}=50$ and different values of $\eta_{\max }$, corresponding to horizontal distances $\mathrm{r}=20,50,200$ and $2000 \mathrm{~m}$. The height of the channel is $8 \mathrm{~km}$ and the height of the observation point $10 \mathrm{~m}$.

Finally, the impact of $\eta_{\max }$ on the fields is shown in Fig. 21, which is relevant to Test T1-A considering $N=50$ and the following two values:

$$
\eta_{\max }=\left\{\begin{array}{l}
\frac{H-z}{r}=159.80 \\
\frac{H-z_{\min }}{r_{\min }}=399.75
\end{array}\right.
$$

As shown, this different choice does not affect at all the final solution. 


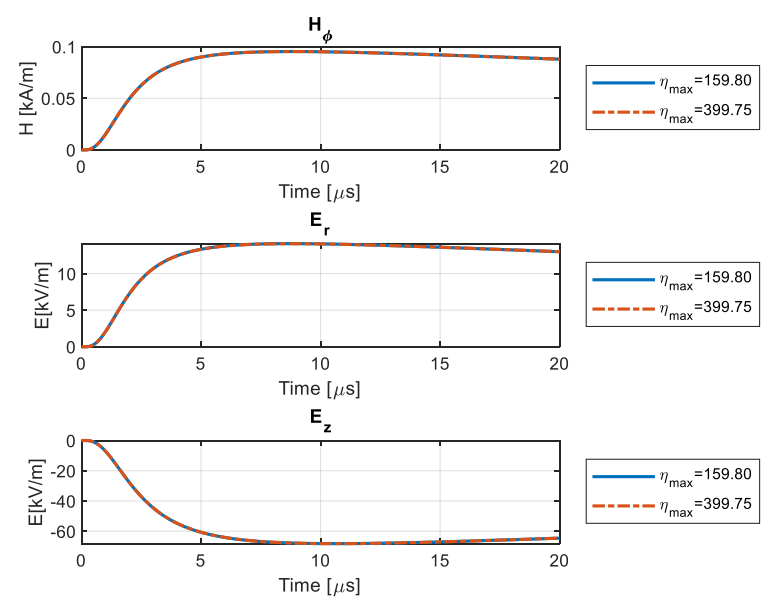

Fig. 21 Test T1-A Comparison with different values of $\eta_{\max }$

\section{B. The choice of $N$}

The computational effort is strictly related to the choice of $N$ since it represents the number of intervals into which the function $f$ is discretized. The bigger $N$, the better the accuracy of the proposed approach, but also the higher the computational effort. An appropriate trade-off between accuracy and computational time has to be found.

In order to determine $N$, the "distance" between $f$ and its piecewise linear approximation $g$ can be computed, minimizing (A6) introduced in the Appendix of Part I. This way, the following function of $N$ is defined

$$
E(N)=\min _{\alpha_{1}, \ldots, \alpha_{N}} \sum_{j=1}^{N+1} H_{j}\left(\frac{\sqrt{1+\alpha_{j}^{2}}-\sqrt{1+\alpha_{j-1}{ }^{2}}}{\sqrt{2\left(\sqrt{1+\alpha_{j}^{2}} \sqrt{1+\alpha_{j-1}^{2}}-\alpha_{j} \alpha_{j-1}-1\right)}}\right)
$$

with $0<\alpha_{1}<\alpha_{2}<\ldots<\alpha_{N}<\eta_{\max }$. The behavior of $E$, as displayed in Fig. 22 is monotonically decreasing. Hence, once a desired threshold for $E$ is introduced, the value of $N$ is automatically fixed.

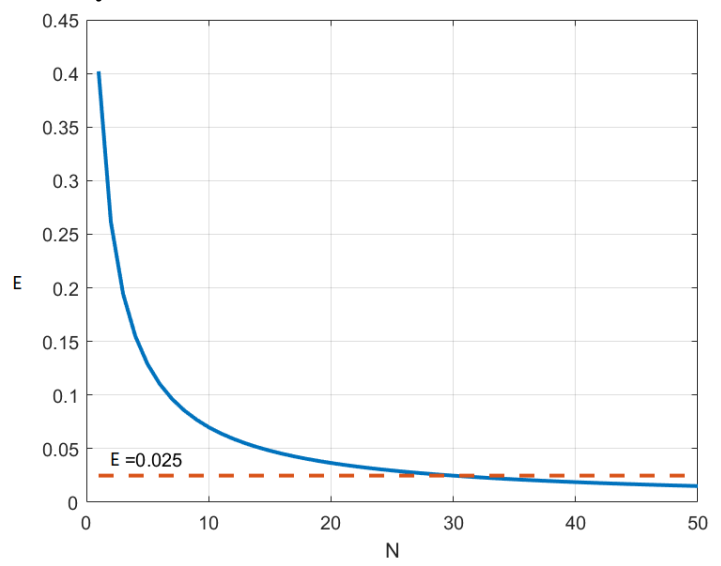

Fig. 22 Behavior of $E$ in (10) as a function of $N$. For a threshold of 0.025 , the corresponding value of $N$ is 30 .

\section{Computational effort of the proposed method}

In the following, attention is focused on the influence of $N$ on the computational effort and on the accuracy of the method.
For each field component $\left(H_{\varphi}, E_{r}, E_{z}\right)$, the deviation $e_{\psi}$ between the proposed approach and the numerical integration is computed as follows:

$$
e_{\psi}=100\left|\frac{\psi_{p}\left(t_{\psi}^{*}\right)-\psi_{n}\left(t_{\psi}^{*}\right)}{\psi_{n}\left(t_{\psi}^{*}\right)}\right|
$$

where $\psi_{p}$ and $\psi_{n}$ are the generic field calculated with the proposed and the numerical approaches, respectively; $t_{\text {end }}$ is the end of the time window and $t_{\psi}^{*} \in\left[0, t_{\text {end }}\right]$ is the time instant s.t. $\left|\psi_{p}(t)-\psi_{n}(t)\right|$ reaches its maximum value.

The computational gain $G_{\tau}$ with respect to the numerical solution is computed as the ratio between the CPU time $\tau_{\text {numerical }}$ required by the numerical method and the time $\tau_{\text {proposed }}$ required by the proposed approach:

$$
G_{\tau}=\frac{\tau_{\text {numerical }}}{\tau_{\text {proposed }}}
$$

$G_{\tau}$ represents how fast the proposed approach is with respect to the numerical solution.

Let us consider Test T2-A and Test T4-A (these two cases have been chosen to account both for a perfect ground conductivity and a finite ground conductivity). TABLE 2/Fig. 22 and TABLE 3/Fig. 23 express $e_{\psi}$ of each field and $G_{\tau}$ as a function of $N$. In the following, it has been chosen to decrease $N$ until one of the fields deviations exceeds $1 \%$.

TABLE 2 Deviation and computational gain as a function of $N-$ Test T2-A

\begin{tabular}{|c|c|c|c|c|}
\hline$N$ & $e_{E r}[\%]$ & $e_{E z}[\%]$ & $e_{H \varphi}[\%]$ & $G_{\tau}$ \\
\hline 50 & 0.075 & 0.079 & 0.003 & 2540 \\
\hline 40 & 0.080 & 0.123 & 0.004 & 3005 \\
\hline 30 & 0.081 & 0.202 & 0.005 & 4113 \\
\hline 20 & 0.085 & 0.411 & 0.052 & 5248 \\
\hline 10 & 0.120 & $\underline{\mathbf{1 . 1 2 1}}$ & 0.142 & 8931 \\
\hline
\end{tabular}

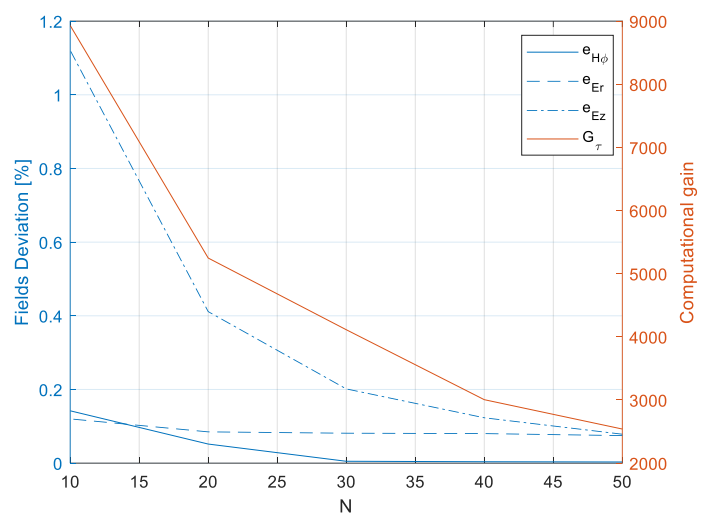

Fig. 23 Deviation and computational gain as a function of $N-$ Test T2-A

TABLE 3 Deviation and computational gain as a function of $N$ - Test T4-A

\begin{tabular}{|c|c|c|c|c|}
\hline$N$ & $e_{E r}[\%]$ & $e_{E z}[\%]$ & $e_{H \varphi}[\%]$ & $G_{\tau}$ \\
\hline 50 & 0.084 & 0.079 & 0.003 & 208 \\
\hline 40 & 0.087 & 0.123 & 0.004 & 224 \\
\hline 30 & 0.089 & 0.202 & 0.005 & 243 \\
\hline 20 & 0.099 & 0.411 & 0.052 & 257 \\
\hline 10 & 0.125 & $\mathbf{1 . 1 2 1}$ & 0.142 & 271 \\
\hline
\end{tabular}




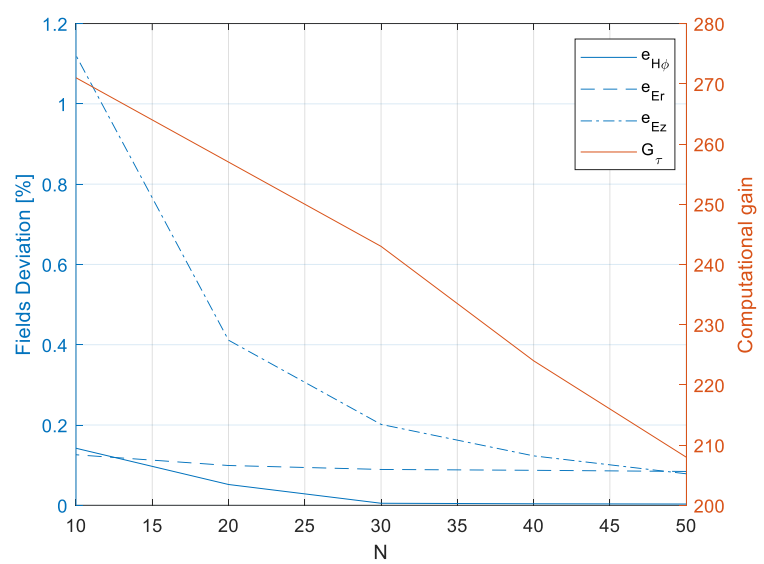

Fig. 24 Deviation and computational gain as a function of $N$ - Test T4-A

As can be noticed, $N$ can be strongly reduced without causing a high increase in the deviation. Moreover, the last columns of TABLE 2 and Fig. 23 underline that even with high values of $N$, the computational time is reduced by a factor of more than 200 , which represents a really impactful advantage of the proposed method. A stronger reduction of the computational time can be obtained in the case of a perfect ground conductor.

In order to provide a comprehensive approach for the reader who wants to evaluate the electromagnetic fields with the approach proposed in this paper, the discretizing points $\alpha_{i}$ and the coefficients $a_{i}, b_{i}$, necessary for the implementation of the proposed procedure, are provided in TABLE 4 with $N=30$, which represents a good compromise between the accuracy and the computational effort gain (note that $\eta_{\max }$ is chosen as in Subsection A).

TABLE 4 Discretizing points $\alpha_{i}$ and coefficients $a_{i}, b_{i}$ with $N=30$

\begin{tabular}{|c|c|c|c|}
\hline $\boldsymbol{i}$ & $\boldsymbol{a}_{\boldsymbol{i}}$ & $\boldsymbol{a}_{\boldsymbol{i}}$ & $\boldsymbol{b}_{\boldsymbol{i}}$ \\
\hline 1 & 0.0815 & 0.0407 & 1 \\
\hline 2 & 0.1639 & 0.1217 & 0.9933 \\
\hline 3 & 0.2479 & 0.2015 & 0.9803 \\
\hline 4 & 0.3344 & 0.2793 & 0.9610 \\
\hline 5 & 0.4245 & 0.3545 & 0.9358 \\
\hline 6 & 0.5191 & 0.4263 & 0.9053 \\
\hline 7 & 0.6196 & 0.4944 & 0.8700 \\
\hline 8 & 0.7273 & 0.5582 & 0.8305 \\
\hline 9 & 0.8440 & 0.6174 & 0.7874 \\
\hline 10 & 0.9717 & 0.6717 & 0.7415 \\
\hline 11 & 1.1129 & 0.7211 & 0.6935 \\
\hline 12 & 1.2707 & 0.7656 & 0.6440 \\
\hline 13 & 1.4490 & 0.8052 & 0.5937 \\
\hline 14 & 1.6528 & 0.8400 & 0.5433 \\
\hline 15 & 1.8884 & 0.8703 & 0.4932 \\
\hline 16 & 2.1643 & 0.8964 & 0.4440 \\
\hline 17 & 2.4918 & 0.9185 & 0.3961 \\
\hline 18 & 2.8863 & 0.9370 & 0.3501 \\
\hline 19 & 3.3690 & 0.9522 & 0.3061 \\
\hline 20 & 3.9704 & 0.9646 & 0.2645 \\
\hline 21 & 4.7350 & 0.9744 & 0.2255 \\
\hline 22 & 5.7300 & 0.9820 & 0.1893 \\
\hline 23 & 7.0614 & 0.9878 & 0.1561 \\
\hline 24 & 8.9026 & 0.9921 & 0.1259 \\
\hline 25 & 11.5559 & 0.9951 & 0.0989 \\
\hline 26 & 15.5829 & 0.9972 & 0.0751 \\
\hline 27 & 22.1294 & 0.9985 & 0.0545 \\
\hline 28 & 33.8382 & 0.9993 & 0.0373 \\
\hline
\end{tabular}

\begin{tabular}{|c|c|c|c|}
\hline 29 & 58.0277 & 0.9997 & 0.0233 \\
\hline $30=N$ & 121.7485 & 0.9999 & 0.0127 \\
\hline $31=N+1$ & 399.750 & 0.9999 & 0.0053 \\
\hline
\end{tabular}

\section{CONCLUSIONS}

The paper presented the validation of the new approach for the computation of lightning electromagnetic fields generated by an arbitrary channel base current over an ideal and a lossy ground proposed in the companion paper.

In the first part, a step-by-step procedure for the implementation of the method described in the companion paper was proposed. In the second part, the validation of the method against the well-known numerical method was shown considering different channel base currents, different ground conductivities and different distances from the stroke location. Finally, the computational effort of the proposed method was analyzed and discussed, underlining the gain in terms of computational time with respect to the traditional numerical method.

\section{REFERENCES}

[1] F. Rachidi, C. A. Nucci, M. Ianoz, and C. Mazzetti, "Influence of a lossy ground on lightning-induced voltages on overhead lines," IEEE Transactions on Electromagnetic Compatibility, vol. 38, pp. 250-264, 1996.

[2] Vernon Cooray, The Lightning Flash: Institution of Engineering and Technology, 2014.

[3] Vernon Cooray, Lightning Electromagnetics: Institution of Engineering and Technology, 2012.

[4] V. Cooray and V. Scuka, "Lightning-induced overvoltages in power lines: validity of various approximations made in overvoltage calculations," IEEE Transactions on Electromagnetic Compatibility, vol. 40, pp. 355363, 1998.

[5] M. Rubinstein, "An approximate formula for the calculation of the horizontal electric field from lightning at close, intermediate, and long range," IEEE Transactions on Electromagnetic Compatibility, vol. 38, pp. 531-535, 1996.

[6] F. Napolitano, "An Analytical Formulation of the Electromagnetic Field Generated by Lightning Return Strokes," IEEE Transactions on Electromagnetic Compatibility, vol. 53, pp. 108-113, 2011.

[7] M. Brignone, F. Delfino, R. Procopio, M. Rossi, and F. Rachidi, "Evaluation of Power System Lightning Performance Part II: Application to an Overhead Distribution Network," IEEE Transactions on Electromagnetic Compatibility, vol. 59, pp. 146-153, 2017.

[8] F. Delfino, R. Procopio, M. Rossi, and F. Rachidi, "Prony Series Representation for the Lightning Channel Base Current," IEEE Transactions on Electromagnetic Compatibility, vol. 54, pp. 308-315, 2012.

[9] F. Delfino, P. Girdinio, R. Procopio, M. Rossi, and F. Rachidi, "TimeDomain Implementation of Cooray-Rubinstein Formula via Convolution Integral and Rational Approximation," IEEE Transactions on Electromagnetic Compatibility, vol. 53, pp. 755-763, 2011.

[10] C. D. Weidman and E. P. Krider, "Submicrosecond risetimes in lightning return-stroke fields," Geophysical Research Letters, vol. 7, pp. 955-958, 1980.

[11]M. Rubinstein and M. A. Uman, "Methods for calculating the electromagnetic fields from a known source distribution: Application to lightning," IEEE Transactions on electromagnetic compatibility, vol. 31, pp. 183-189, 1989.

[12] C. F. Barbosa and J. O. S. Paulino, "On the Measurement of Lightning Horizontal Electric Fields," IEEE Transactions on Electromagnetic Compatibility, vol. 61, pp. 681-688, 2019.

[13] H. Heidler, "Analytische Blitzstromfunktion zur LEMP-Berechnung," 18th ICLP, Munich, Germany, 1985.

[14] F. Heidler, J. M. Cvetic, and B. V. Stanic, "Calculation of lightning current parameters," IEEE Transactions on Power Delivery, vol. 14, pp. 399-404, 1999. 